patterns or STI transmission risk. Given this context, we explored the social relevance, understandings and meanings of contemporary sexual partner types, as a first step in aligning lived realities with clinical practice to improve PN outcomes.

Methods We conducted eleven semi-structured focus groups (November 2016-August 2017), with members of the public $(n=38)$ and sexual health clinic attendees diagnosed with an STI in the past six months $(n=19)$ in England and Scotland. We recruited participants aged 18-65 years who identified as heterosexual or men who have sex with men (MSM), using purposive and convenience sampling. Data were digitally recorded, transcribed and analysed using thematic analysis in NVivo V.10.

Results Findings from the 57 participants (male $n=34$; female $\mathrm{n}=23$ ), suggested two key themes in understanding sexual partner types: 1) nature of emotional involvement with the partner(s) and 2) time/continuity of the relationship. Both tapped into participants' relationship perspectives and shaped their understandings and use of partner terms. Interrelated subthemes involved: the different contexts, such as clinical consultations or everyday social interactions, which shaped the use of the terms 'regular' and 'casual' and associated interpretations; and the polysemy and ambiguity of the terms when they were used in combination with other words (e.g. casual sex; casual partner; casual regular) and alternate terms (e.g. random, one-off, serious relationship). There were no differences in the understandings of the terms between heterosexual and MSM participants.

Conclusion This is the first empirical evidence that challenges and provides insight into the dichotomy of sexual partner types in contemporary clinical practice. There is a need for a new socially informed, interdisciplinary classification of sexual partner types to enable better recording and communication between patients, sex partners and healthcare professionals. Improved understanding of partner types will help healthcare professionals develop and tailor PN approaches which address social and cultural influences on the way people form sexual relationships and talk about sex. This will enable targeting of resources to achieve greatest benefit to individual and population health by detecting and preventing STI transmission.

\section{OP32 TRENDS IN USE OF PRESCRIBED MEDICINES BY BODY MASS INDEX AND AGE: EVIDENCE FROM THE LAST TWO DECADES USING HEALTH SURVEYS FOR ENGLAND DATA}

S Scholes, A Solar, JS Mindell*. Epidemiology and Public Health, University College London, London, UK

\subsection{6/jech-2018-SSMabstracts.32}

Background Prescribing is the most common clinical intervention in the NHS, with annual costs exceeding $£ 9$ billion. Understanding differences in patterns of prescribed medicine use over time informs clinical practice and epidemiological research. We compare trends in prescribed medicine use by body mass index (BMI) and age to better understand the factors associated with increased prescribing.

Methods Repeated cross-sectional analysis of nationally-representative Health Surveys for England 1994-2015 ( $\mathrm{n=42} 216$ participants aged $20+$ years with measured BMI and medicine use data). Sex-specific logistic regression models with main effects and interactions between BMI (reference: normal- weight; obese: BMI $\geq 30 \mathrm{~kg} / \mathrm{m}^{2}$ ), age, and survey year on taking any prescribed medicine in the last week (excluding smoking cessation products and contraception) were assessed adjusting for smoking and education. Analyses were repeated for polypharmacy $(3+$ medicines $)$, and for cardiovascular and non-cardiovascular medicines. Results are presented as fullyadjusted Odds Ratios (OR) with 95\% Confidence Intervals (95\% CIs).

Results Overall, the age-standardised prevalence of prescribed medicine use between 1994 and 2015 increased from 37.8\% (95\% CI $36.7 \%$ to $39.0 \%)$ to $46.7 \%(45.2 \%-48.2 \%)$ in men and from $45.7 \%(44.6 \%-46.8 \%)$ to $53.2 \%(51.8 \%-54.6 \%)$ in women. By 2015, use of $3+$ medicines had doubled to $24.6 \%$ $(23.4 \%-25.8 \%)$ in men and to $27.2 \%(26.1 \%-28.3 \%)$ in women. Among those taking any medicine, polypharmacy rose by 1.7 times to $42.8 \%(39.9 \%-45.6 \%)$ and $45.1 \%(42.9 \%-$ $47.2 \%)$ respectively.

Prescribed medicine use increased over time more sharply with age. However, after age-adjustment, the increase in prevalence over time was greatest in obese women (BMI-by-year interaction: $p=0.003)$. The odds of obese women taking any prescribed medicine in the last week were 1.5 times higher than those for normal-weight women in 1994 (OR: 1.49; $95 \% \mathrm{CI} 1.28$ to 1.73 ), but had increased to 2.1 in 2015 $(2.14 ; 1.82-2.53)$. Increased medicine use over time was greatest in obese men for cardiovascular medicines (BMI-by-year interaction: $\mathrm{p}=0.036)$. The odds of obese men aged 50-59 years taking any prescribed cardiovascular medicine in the last week were 2.1 times higher than those for normal-weight men of the same age in $1994(2.08 ; 1.46-2.95)$. The equivalent odds had increased to 3.0 in 2015 (2.98; 2.10-4.21).

Conclusion Higher BMI is associated with increased prescribing over the last 20 years regardless of age, reflecting secular rises in levels of awareness and of treatment of obesity and other co-morbid conditions, such as hypertension and diabetes, increased availability of effective secondary prevention medicines (e.g. statins, ACE inhibitors), lower thresholds for their use, and greater adherence to guidelines for their prescription.

\section{OP33 PERMANENT CHILDHOOD HEARING IMPAIRMENT DETECTED THROUGH UNIVERSAL NEWBORN HEARING SCREENING: SYSTEMATIC REVIEW AND META- ANALYSIS OF PREVALENCE AND SCREENING PROGRAMME PERFORMANCE IN 1.8 MILLION INFANTS}

${ }^{1} \mathrm{E}$ Butcher*, ${ }^{2} \mathrm{C}$ Dezateux, ${ }^{3} \mathrm{M}$ Cortina-Borja, ${ }^{1} \mathrm{R}$ Knowles. 'Life Course Epidemiology and Biostatistics, University College London Great Ormond Street Institute of Child Health, London, UK: ${ }^{2}$ Centre for Primary Care and Public Health, Queen Mary University of London, London, UK; ${ }^{3}$ Clinical Epidemiology, Nutrition and Biostatistics, University College London Great Ormond Street Institute of Child Health, London, UK

\subsection{6/jech-2018-SSMabstracts.33}

Background Systematic appraisal of universal newborn hearing screening (UNHS) programme performance and the prevalence of permanent childhood hearing impairment (PCHI) detected is lacking, including for those admitted to Neonatal Intensive Care Units (NICU). We carried out a systematic review and meta-analysis of studies reporting PCHI prevalence (defined as bilateral loss $\geq 26 \mathrm{~dB} \mathrm{HL}$ ) detected through UNHS (defined as universal screening using otoacoustic emissions and/or auditory brainstem response testing by age 6 months) in very-highly developed countries (PROSPERO:CRD42016051267). We 
estimated screening test performance and PCHI prevalence in those who were or were not admitted to NICU.

Methods Eligible studies, reporting UNHS-detected PCHI prevalence in very highly-developed countries (no restrictions by language or date), were identified from six electronic databases (January 2017) along with references of cited papers and unpublished literature (November 2017). Papers reporting on at-risk populations only, with no English abstract (unless unpublished), or of ineligible study type were excluded. Two reviewers independently extracted data and assessed quality of included papers using criteria adapted from the NewcastleOttawa, STARD and QUADAS-2 tools, with differences resolved by consensus. Pooled prevalence was estimated from random-effects models using Freeman-Tukey double arcsine transformation. Negative predictive value (NPV), sensitivity and specificity were calculated only for studies with follow-up to ascertain false negatives, whilst positive predictive value (PPV) calculation was not restricted by follow-up. Confidence intervals $(95 \% \mathrm{CI})$ were estimated using Wilson (Score) methods (Stata: Release 15; StataCorp LP).

Results 41 eligible reports on 32 study populations $(1,799,863$ infants) were identified from 6195 non-duplicate references. Pooled UNHS-detected PCHI prevalence was 1.08 (95\% CI 0.90 to 1.28$)$ per 1000 screened $\left(I^{2}=89.2 \%\right)$. Prevalence was 6.9 times (95\% CI 3.8 to 12.5 ) higher among those admitted to NICU (3 studies). Smaller studies were significantly associated with larger prevalences (Egger's test: $p=0.017)$. PPV ranged from 1.5\%-83.5\% (25 studies), NPV 100\% (7 studies), sensitivities 88.9\%-100\% (8 studies) and specificities 92.3\%$99.9 \%$ (7 studies). Quantitative pooling of screening programme performance was not possible due to methodological differences.

Conclusion In very highly-developed countries, around 1 per 1000 screened infants will require PCHI investigation and management. Prevalence is almost 7 times higher in infants admitted to NICU. Strengths of our study include the systematic search strategy and robust statistical methods. Our findings are limited to very highly-developed countries. Estimates were restricted by lack of high-quality reporting on attrition and surveillance. Improved reporting of surveillance and attrition should be encouraged to enable evaluation of screening programme performance.

ESRC-funded PhD ES/J500185/1.

\section{School-based interventions}

\section{OP34 \#THE PRIMARY OUTCOMES OF THE SOCIAL AND EMOTIONAL EDUCATION AND DEVELOPMENT (SEED) TRIAL: A STRATIFIED, CLUSTER RANDOMISED TRIAL OF A MULTI-COMPONENT PRIMARY SCHOOL INTERVENTION IN SCOTLAND AIMED AT IMPROVING PUPILS' SOCIAL AND EMOTIONAL WELLBEING}

\begin{abstract}
${ }^{1} \mathrm{MM}$ Henderson*, 'S Tweedie, ${ }^{2} \mathrm{~K}$ Wetherall, ${ }^{2} \mathrm{~A}$ McConnachie, ${ }^{3} \mathrm{~K}$ Levin, ${ }^{4} \mathrm{P}$ Wilson, ${ }^{1} S$ Smillie, ${ }^{1} \mathrm{D}$ Wight. ${ }^{1} \mathrm{MRC/CSO}$ Social and Public Health Sciences Unit (SPHSU), University of Glasgow, Glasgow, UK; ${ }^{2}$ Robertson Centre for Biostats, University of Glasgow, Glasgow, UK; ${ }^{3}$ Glasgow Royal Infirmary, NHS Greater Glasgow and Clyde, Glasgow, UK; ${ }^{4}$ Centre for Rural Health, University of Aberdeen, Aberdeen, UK
\end{abstract}

10.1136/jech-2018-SSMabstracts.34

Background The Social and Emotional Education and Development (SEED) intervention process aimed to improve the social and emotional wellbeing (SEW) of primary school pupils. The iterative process involved three components: 1 . questionnaire completion: 2. providing benchmarked feedback to all staff; and 3. All staff involved in reflexive discussion, led by educational psychologists, to facilitate selection and implementation of evidence-based initiatives (resource guide provided) to address pupils' SEW needs.

Methods A stratified randomised controlled trial involved 37 schools across Scotland and was conducted between 2013 and 2018. This involved 2639 pupils across two cohorts. At baseline the younger cohort were aged 4-5 and the older cohort were aged 8-9. After a one year gap, to enable commencement of action plans, three waves of follow-up data were collected annually. The primary outcome was the Total Difficulties score from the Strengths and Difficulties Questionnaire (SDQ) at Follow-up 3, when the younger pupils were aged $8-9$ and the older pupils were aged 12-13. Secondary outcomes included all five SDQ subscales.

Hierarchical regression analysis allowing for clustering at school learning community level was conducted in the statistical package, 'R'. Missing data was handled using repeated measures.

Results The primary outcome, pupils' SDQ Total Difficulties at Follow-up 3, showed a statistically significant result in the desired direction: $-1.334(-1.918,-0.751), \mathrm{p}<0.001$. (Please note these are preliminary results and are still to be formally published, whilst robust, final figures may vary slightly after reviewers' comments). All five SDQ subscales also showed beneficial and statistically significant results.

Subgroup analysis showed that all results were stronger for the older cohort, particularly the older boys. The results were significant for both affluent and deprived pupils.

Discussion The SEED intervention process led to beneficial results for the social and emotional wellbeing of intervention schools' pupils. The Scottish Government are actively planning a SEED type of process for Scotland, we hope to extend that throughout the UK. Longer term outcomes can be explored using routine data.

\section{OP35 A PROCESS EVALUATION OF THE SOCIAL AND EMOTIONAL EDUCATION AND DEVELOPMENT TRIAL: CASE STUDY FINDINGS}

${ }^{1} S$ Tweedie*, ${ }^{1} S$ Smillie, ${ }^{1} D$ Wight, ${ }^{2} \mathrm{~L}$ Elliott, ${ }^{1} \mathrm{C}$ Purcell, ${ }^{3} \mathrm{~S}$ Haw, ${ }^{4} \mathrm{~L}$ Bond, ${ }^{1} \mathrm{M}$ Henderson. ${ }^{1} \mathrm{MRC/CSO}$ Social and Public Health Sciences Unit, University of Glasgow, Glasgow, UK; ${ }^{2}$ Health and Life Sciences, Glasgow Caledonian University, Glasgow, UK; ${ }^{3}$ Health Sciences, University of Stirling, Stirling, UK; ${ }^{4}$ The Victoria Institute, Victoria University, Melbourne, Australia

\subsection{6/jech-2018-SSMabstracts.35}

Background The Social and Emotional Education and Development programme (SEED) aims to improve the social and emotional wellbeing (SEW) of primary school pupils. It is based on a cycle of collecting school specific data on pupil and staff SEW, providing benchmarked feedback and facilitating the adoption of evidence-based initiatives to address need.

A stratified randomised controlled trial (RCT) involved 37 schools across three local authority districts in Scotland between 2013 and 2017. Complementing the RCT was a detailed process evaluation, which enabled interpretation of the trial outcomes and answered secondary questions on implementation, mechanisms and context. We present the first stage of the process evaluation, an assessment of the likely 